\title{
In vitro behaviour of curcumin nanoemulsions stabilized by biopolymer emulsifiers - Effect of interfacial composition
}

\author{
Ana C. Pinheiro ${ }^{\text {a, }}$, Manuel A. Coimbra ${ }^{\text {b }}$, António A. Vicente ${ }^{\text {a }}$ \\ a CEB, Centre of Biological Engineering, University of Minho, 4710-057 Braga, Portugal \\ ${ }^{\mathrm{b}}$ QOPNA, Department of Chemistry, University of Aveiro, 3810-193 Aveiro, Portugal
}

\section{A R T I C L E I N F O}

\section{Article history:}

Received 15 May 2015

Received in revised form

23 July 2015

Accepted 25 July 2015

Available online 29 July 2015

\section{Keywords:}

Multilayer nanoemulsions

Lactoferrin

Alginate

In vitro digestion

Dynamic gastrointestinal system

\begin{abstract}
A B S T R A C T
Nanoemulsions present a high potential to be used in food products due to their advantages over conventional emulsions (e.g. higher stability to gravitational separation and droplet aggregation and enhanced bioavailability of encapsulated compounds), however their application to foods is hindered by some concerns about potential risks associated with their ingestion. The knowledge of the behaviour of nanoemulsions as well as the fate of bioactive compounds encapsulated within them in the gastrointestinal (GI) tract is of utmost importance to assess their safety for human consumption and to produce delivery systems that provide an optimized bioactivity of the encapsulated compound. In this work, a dynamic gastrointestinal model, comprising the simulation of stomach, duodenum, jejunum and ileum, was used to evaluate the behaviour of curcumin nanoemulsions stabilized by biopolymer emulsifiers (lactoferrin and lactoferrin/alginate multilayer structure) under GI conditions. The interfacial characteristics of curcumin nanoemulsions had a significant impact on their physicochemical stability within the simulated GI tract. Also, results suggested that alginate coating may be able to control the rate of lipid digestion and free fatty acids adsorption within the GI tract, but the encapsulated lipid is digested at the same extent, releasing the lipophilic bioactive compound. This work contributes to an improved understanding of how multilayer nanoemulsions behave within the GI tract and this knowledge will be useful for the optimization of delivery systems that improve the physicochemical stability of emulsions in food products, while still releasing encapsulated lipophilic bioactive compounds.
\end{abstract}

() 2015 Elsevier Ltd. All rights reserved.

\section{Introduction}

The growing consumers' awareness of the link between food and health and the consequent increase in demand for functional foods are stimulating the innovation and the development of new products in the food industry. Many health-promoting compounds are lipophilic (e.g. curcumin, $\omega-3$ fatty acids, lycopene, phytosterols, quercetin, resveratrol), however their utilization by food industry is limited due to their low bioavailability and difficulties associated with their incorporation into food matrices (McClements \& Xiao, 2012). In particular, curcumin (a natural polyphenolic phytochemical extracted from the powdered rhizomes of turmeric spice) has been reported to have many biological activities, such as antitumor, anti-oxidant, anti-microbial and anti-inflammatory properties. However, its application as a

\footnotetext{
* Corresponding author.

E-mail address: anapinheiro@deb.uminho.pt (A.C. Pinheiro).
}

bioactive ingredient is currently limited by its poor water solubility (hindering its incorporation into many food products) and low bioavailability (which means that its health-promotion effects may not be effective) (Ahmed, Li, McClements, \& Xiao, 2012). Emulsion-based delivery systems are one of the vehicles that are being increasingly used to both protect and deliver lipophilic bioactive compounds (increasing their bioavailability) and to achieve their incorporation into food products (Salminen, Herrmann, \& Weiss, 2013). These systems can be produced entirely from bio-based materials, using simple processing operations such as blending or homogenization (Tokle \& McClements, 2011). For example, lactoferrin, a globular protein derived from milk with a wide range of reported biological activities (e.g. antioxidant antimicrobial, cancer prevention, and modulation of immune responses) can be used as natural emulsifier to enhance the formation and stabilization of oil-in-water emulsions (Balcão et al., 2013; Shimoni, Shani Levi, Levi Tal, \& Lesmes, 2013).

The design of emulsion-based delivery systems to control digestion, release and absorption of encapsulated lipophilic 
bioactive compounds within the GI tract is one of the current challenges of food industry. Digestibility of lipids and release of bioactive lipophilic compounds encapsulated within emulsionbased delivery systems can be controlled by coating lipid droplets with biopolymer coatings. These multilayer emulsions can be formed by electrostatic deposition of ionic biopolymers onto oppositely charged lipid droplets (layer-by-layer (LbL) technique) (Decher \& Schlenoff, 2003; Li et al., 2010). Alginate, a naturally occurring anionic polysaccharide typically obtained from brown seaweed, is one of the polyelectrolytes most used for LbL deposition (Maurstad, Mørch, Bausch, \& Stokke, 2008; Zhou et al., 2010), which is probably due to its biocompatibility, low toxicity and relatively low cost (Lee \& Mooney, 2012). The design of the multilayer coatings interface will depend on their specific application: multilayer coatings can be used to increase the bioavailability of incorporated bioactive compounds, being in this case essential that it is broken down within the GI tract so that the bioactive compounds can be released and absorbed. It can also be designed to be completely indigestible within the GI tract, when used to reduce the caloric content of foods by preventing lipases from accessing encapsulated lipids (McClements, 2010).

There is a wide range of emulsion-based delivery systems that can be assembled entirely from bio-based materials. Nanoemulsions (radius $<100 \mathrm{~nm}$ ) present advantages over conventional emulsions, such as higher stability to gravitational separation and droplet aggregation, higher optical clarity and enhanced bioavailability of encapsulated lipophilic bioactive compounds (due to their small size) (McClements \& Xiao, 2012). Despite of the potential benefits of nanoemulsions, their application to foods is hindered by some concerns about potential risks associated with the ingestion of nanostructures, such as the potential toxicity of some components used to produce them (e.g. surfactants and organic solvents) and their ability to alter the biological fate of ingested materials and encapsulated bioactive compounds, which could potentially have some adverse effects on human health (McClements, 2013). The first issue can be solved by using only bio-based food grade materials when producing nanoemulsions; the second issue is a broader one and must be evaluated (ideally) in vivo, or at least in vitro. The knowledge of the behaviour of nanoemulsions as well as the fate of bioactive compounds encapsulated within them in the GI tract is of utmost importance to either assess their safety for human consumption and to produce tailored delivery systems (i.e. with optimized bioactivity). In recent years, considerable efforts have been made in understanding the physicochemical behaviour of nanoemulsions within the GI tract (Ahmed et al., 2012; Salvia-Trujillo, Qian, Martín-Belloso, \& McClements, 2013), however further work is clearly needed because there is still a lack of knowledge regarding the major factors that influence the biological fate of ingested nanoemulsions. Also, most of the works that evaluate the behaviour of emulsions under gastrointestinal conditions use static in vitro digestion models and simplified gastrointestinal conditions that do not accurately simulate the complex physicochemical and physiological processes that occur within the human gastrointestinal tract (Li et al., 2010; Mun, Decker, \& McClements, 2007). Therefore, being very difficult (often infeasible) the use of in vivo models (high costs and ethical constrains often involved), there is a need of using more realistic in vitro gastrointestinal models.

In this work, a dynamic digestion model comprising the simulation of stomach, duodenum, jejunum and ileum, has been used to evaluate the behaviour of curcumin nanoemulsions stabilized by biopolymer emulsifiers (lactoferrin and lactoferrin/alginate multilayer structure) under gastrointestinal conditions.

\section{Materials and methods}

\subsection{Materials}

Corn oil (Fula) was purchased from a local supermarket in Braga (Portugal) and was used without further purification. Curcumin was purchased from Sigma-Aldrich (St. Louis, MO), lactoferrin was acquired from DMV International (Veghel, The Netherlands) and sodium alginate was purchased from Kelco International, Ltd. (UK). Pepsin from porcine gastric mucosa $\left(600 \mathrm{U} \mathrm{mL}^{-1}\right)$, lipase from porcine pancreas $\left(40 \mathrm{U} \mathrm{mL}^{-1}\right)$, pancreatin from porcine pancreas $(8 \times$ USP), bile extract porcine and the salts used for preparing the gastric and small intestinal electrolyte solutions, hydrochloric acid and sodium bicarbonate were purchased from Sigma-Aldrich (St. Louis, MO). Chloroform was obtained from Fisher Scientific (NJ, USA), acetone from Fisher Chemical (UK) and sodium hydroxide and phenolphthalein from Panreac (Spain).

\subsection{Emulsion preparation}

Curcumin nanoemulsions (stabilized by lactoferrin) were prepared by homogenizing $5 \mathrm{wt} . \%$ corn oil containing $0.1 \mathrm{wt}$.\% of curcumin with 95 wt.\% aqueous emulsifier solution (lactoferrin at a concentration of $2 \%$ ), using an Ultra-Turrax homogenizer (T 25, IkaWerke, Germany) for 2 min followed by passage through a high pressure homogenizer (NanoDeBee, Bee International, South Easton, Massachusetts, USA) at 20,000 Psi (137.9 MPa), for 20 cycles.

Curcumin multilayer nanoemulsions (stabilized by lactoferrin/ alginate multilayer structure) were prepared by adding the curcumin nanoemulsion ( $\mathrm{pH} \mathrm{4}$, at which lactoferrin has the maximum charge) dropwise with a syringe pump (NE-1000, New Era Pump Systems, Inc., USA) to an alginate solution ( $\mathrm{pH} \mathrm{7,} \mathrm{at} \mathrm{which} \mathrm{alginate}$ has the maximum charge) under stirring for $30 \mathrm{~min}$. The multilayer nanoemulsion was then treated with sonication (during 2 min at a frequency of $50-60 \mathrm{kHz}$ ) (Brason 5510, Bransonic ${ }^{\circledR}$ ultrasonic cleaner, USA) to disrupt any flocculated droplets formed during its preparation (Li et al., 2010). Different concentrations of alginate were tested in order to optimize the formulation. The final $\mathrm{pH}$ of the multilayer nanoemulsions was around 5.

Both nanoemulsions were kept at $4{ }^{\circ} \mathrm{C}$ in the dark before characterization and in vitro digestion.

\subsection{In vitro digestion}

\subsubsection{Gastrointestinal model}

A dynamic gastrointestinal system was used in the in vitro digestion experiments. This model simulates the main events that occur during digestion and consists of four compartments simulating the stomach, duodenum, jejunum and ileum. Each compartment consists in two connected glass reactors with a flexible wall inside and water is pumped around the flexible walls to maintain the temperature at $37^{\circ} \mathrm{C}$ and to enable the simulation of the peristaltic movements (by the alternate compression and relaxation of the flexible walls). The changes in water pressure are achieved by peristaltic pumps which alter the flow direction according to the time controlling devices connected to them. The compartments are connected by silicone tubes and, at a predefined time, a constant volume of chyme is transferred. All compartments are equipped with $\mathrm{pH}$ electrodes and $\mathrm{pH}$ values are controlled by the secretion of $\mathrm{HCl}\left(1 \mathrm{~mol} \mathrm{~L}^{-1}\right)$ into the stomach and $\mathrm{NaHCO}_{3}\left(1 \mathrm{~mol} \mathrm{~L}^{-1}\right)$ into the intestinal compartments. The gastric and intestinal secretions are added via syringe pumps at pre-set flow rates. The jejunum and ileum compartments are connected with hollow-fibre devices (SpectrumLabs Minikros ${ }^{\circledR}$, M20S-100- 
01P, USA) to absorb digestion products and water from the chyme and to modify electrolyte and bile salts concentration of the chyme (Reis et al., 2008).

It should be noted that, although at the mouth stage there may be changes in emulsions' size and interfacial characteristics, influencing the emulsions' fate in the GI tract (McClements et al., 2012), this phase was not included once the sample is liquid (and therefore the mastication is not relevant and the residence time in the mouth is very low) and the samples do not contain starch (i.e. the primary enzyme present in saliva, amylase, would not act).

\subsubsection{Experimental conditions}

In vitro digestion was performed as described by other authors (Reis et al., 2008) with some modifications. A volume of $60 \mathrm{~mL}$ of curcumin nanoemulsions (both nanoemulsions and multilayer nanoemulsions) was introduced into the dynamic gastrointestinal system (gastric compartment) and the experiment was run for a total of $5 \mathrm{~h}$, simulating average physiological conditions of GI tract by the continuous addition of gastric, duodenal, jejunal and ileal secretions. The gastric secretion consisted of pepsin and lipase in a gastric electrolyte solution $\left(\mathrm{NaCl} 4.8 \mathrm{~g} \mathrm{~L}^{-1}, \mathrm{KCl} 2.2 \mathrm{~g} \mathrm{~L}^{-1}\right.$, $\mathrm{CaCl}_{2} 0.22 \mathrm{~g} \mathrm{~L}^{-1}$ and $\mathrm{NaHCO}_{3} 1.5 \mathrm{~g} \mathrm{~L}^{-1}$ ), secreted at a flow rate of $0.33 \mathrm{~mL} \mathrm{~min}{ }^{-1}$. The $\mathrm{pH}$ was controlled to follow a predetermined curve (from 4.8 at $t=0$ to 1.7 at $t=120 \mathrm{~min}$ ) by secreting $\mathrm{HCl}\left(1 \mathrm{~mol} \mathrm{~L}^{-1}\right)$.

The duodenal secretion consisted of a mixture of $4 \%(w / v)$ porcine bile extract, $7 \%(\mathrm{w} / \mathrm{v})$ pancreatin solution and small intestinal electrolyte solution (SIES) $\left(\mathrm{NaCl} 5 \mathrm{~g} \mathrm{~L}^{-1}, \mathrm{KCl} 0.6 \mathrm{~g} \mathrm{~L}^{-1}\right.$, $\mathrm{CaCl}_{2} 0.25 \mathrm{~g} \mathrm{~L}^{-1}$ ) secreted at a flow rate of $0.66 \mathrm{~mL} \mathrm{~min}^{-1}$. The jejunal secretion fluid consisted of SIES containing $10 \%(\mathrm{v} / \mathrm{v})$ porcine bile extract solution at a flow rate of $2.13 \mathrm{~mL} \mathrm{~min}^{-1}$. The ileal secretion fluid consisted of SIES at a flow rate of $2.0 \mathrm{~mL} \mathrm{~min}^{-1}$. The $\mathrm{pH}$ in the different parts of small intestine was controlled by the addition of $1 \mathrm{~mol} \mathrm{~L}^{-1} \mathrm{NaHCO}_{3}$ solution to set-points of 6.5, 6.8 and 7.2 for simulated duodenum, jejunum and ileum, respectively. During in vitro digestion, samples were collected directly from the lumen of the different compartments, from the jejunal and ileal filtrates and from the ileal delivery. The jejunal and ileal filtrates were used to determine the bioaccessible fraction of curcumin. The samples were analysed for size, $\zeta$-potential and free fatty acids. Both curcumin nanoemulsions were tested in the dynamic gastrointestinal model at least in triplicate.

\subsection{Particle size}

The particle size of the emulsions was measured at different stages of digestion by dynamic light scattering (Zetasizer Nano ZS, Malvern Instruments, Worcestershire, UK). The Z-average mean diameter was calculated by the instrument using Stokes-Einstein equation, assuming that nanoemulsion droplets were spherical. Before measurement, the samples were diluted at a ratio of $1: 100(\mathrm{v} / \mathrm{v})$ in an appropriate buffer solution with the same $\mathrm{pH}$ as the aqueous phase of the sample $\left(\mathrm{KCl}-\mathrm{HCl}, 10 \mathrm{mmol} \mathrm{L}^{-1}, \mathrm{pH} 2\right)$ for stomach sample and phosphate buffer $\left(10 \mathrm{mmol} \mathrm{L}^{-1}, \mathrm{pH}\right.$ 7) for initial and small intestine samples (Hur, Decker, \& McClements, 2009) at room temperature to avoid multiple scattering effects. The measurements were performed in triplicate.

\section{5. ऊ-potential}

The $\zeta$-potential values of nanoemulsions at different stages of digestion were measured using a particle electrophoresis instrument (Malvern Instruments, Worcestershire, UK). The $\zeta$-potential was determined by measuring the direction and velocity of droplet movement in the applied electric field and was calculated by the instrument using the Smoluchowski model.

The samples were diluted at a ratio of $1: 100(\mathrm{v} / \mathrm{v})$ in appropriate buffer solution ( $\mathrm{KCl}-\mathrm{HCl}, 10 \mathrm{mmol} \mathrm{L}^{-1}, \mathrm{pH} 2$ ) for stomach sample and phosphate buffer ( $\left.10 \mathrm{mmol} \mathrm{L}^{-1}, \mathrm{pH} 7\right)$ for initial and small intestine samples (Hur et al., 2009) at room temperature before measurement. Each individual $\zeta$-potential measurement was determined from the average of three readings.

\subsection{Transmission electron microscopy (TEM)}

TEM micrographs were conducted on a Zeiss EM 902 A microscope (Germany) at accelerating voltages of $50 \mathrm{kV}$ and $80 \mathrm{kV}$. The samples were prepared by dropping nanoemulsion solutions onto copper grids coated with carbon film, followed by staining with uranyl acetate and natural drying at room temperature.

\subsection{Free fatty acids released}

The digestion activity was measured by determining the amount of free fatty acids (FFA) released from curcumin nanoemulsions using a titration method (Pinsirodom, 2005). Briefly, $5 \mathrm{~mL}$ of jejunal filtrate, ileal filtrate and ileal delivery samples were collected and $10 \mathrm{~mL}$ of acetone were added to quench the enzymes' activity and 3 drops of $1 \%(\mathrm{w} / \mathrm{v})$ phenolphthalein were added as an indicator. A direct titration with $0.1 \mathrm{~mol} \mathrm{~L}^{-1} \mathrm{NaOH}$ using a burette was performed and the volume of $\mathrm{NaOH}$ added until the titration end point was determined and used to calculate the concentration of FFA produced by lipolysis. Therefore, the percentage of free fatty acids released was calculated from the number of moles of $\mathrm{NaOH}$ required to neutralize the FFA divided by the number of moles of FFA that could be produced from triglycerides if they were all digested (assuming 2 FFA produced per 1 triacylglycerol molecule) (Li, Hu, Du, Xiao, \& McClements, 2011):

$$
\% \text { FFA }=100 \times\left(\frac{v_{\text {NaOH }} \times m_{\text {NaOH }} \times M_{\text {lipid }}}{w_{\text {lipid }} \times 2}\right)
$$

where $v_{\mathrm{NaOH}}$ is the volume of sodium hydroxide required to neutralize the FFA generated (in $\mathrm{mL}$ ), $m_{\mathrm{NaOH}}$ is the molarity of the sodium hydroxide used (in mol L ${ }^{-1}$ ), $w_{\text {lipids }}$ is the total weight of corn oil initially present and $M_{\text {lipid }}$ is the molecular weight of the corn oil (based on their average fatty acid composition the molecular weight of corn oil was considered to be $800 \mathrm{~g} \mathrm{~mol}^{-1}$ ).

Once the carboxylic groups of alginate presented in the multilayer nanoemulsions can act as a buffer, interfering with the FFA quantification, a blank assay was performed only with alginate.

\subsection{Curcumin bioaccessibility}

It was assumed that the fraction of the original curcumin that ended up in the micelle phase was a measure of curcumin bioaccessibility (Ahmed et al., 2012) and that the mixed micelles that contained the bioavailable curcumin fraction were able to pass the hollow-fibre membranes (i.e. corresponds to jejunal filtrate and ileal filtrate samples), while undigested emulsions were retained (Minekus et al., 2005). Curcumin bioaccessibility was determined based on the methodology described by other authors (Ahmed et al., 2012). Briefly, $5 \mathrm{~mL}$ of the sample (jejunal or ileal filtrate) were vortexed with $5 \mathrm{~mL}$ of chloroform, and then centrifuged (Sigma $4 \mathrm{~K} 15$, Germany) at $1750 \mathrm{rpm}$, at room temperature, for $10 \mathrm{~min}$. The bottom chloroform layer was collected and the extraction procedure was repeated with the top layer. The second bottom chloroform layer was added to the previously set aside chloroform layer, mixed, and analysed in a UV-VIS spectrophotometer (Jasco V560, USA) at 
a)

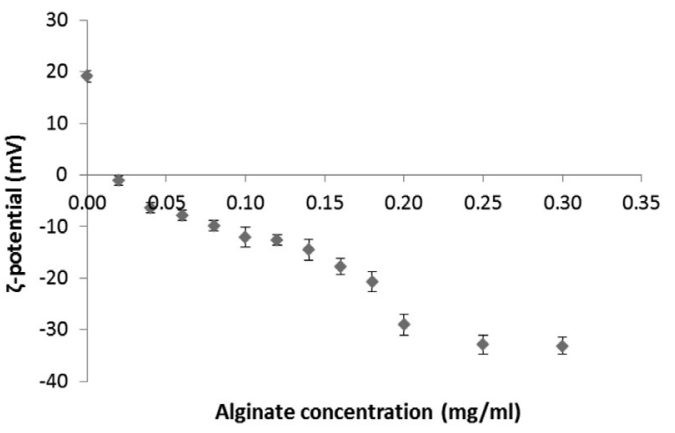

b)

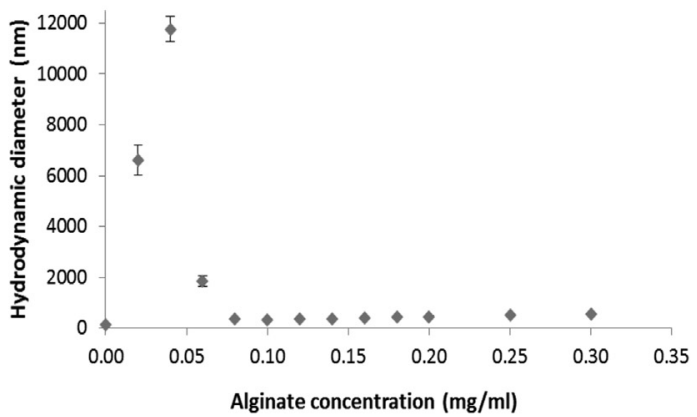

Fig. 1. Influence of alginate concentration on the $\zeta$-potential (a) and size (b) of multilayer nanoemulsions.

$419 \mathrm{~nm}$ (absorbance peak). The concentration of curcumin was determined from a previously prepared calibration curve of absorbance versus curcumin concentration in chloroform.

\subsection{Statistical analyses}

All experiments were carried out at least in triplicate using freshly prepared samples. The results were then reported as averages and standard deviations of these measurements. The statistical analyses were carried out using analysis of variance, Tukey mean comparison test $(p<0.05)$ and linear regression analysis (SigmaStat, trial version, 2003, USA).

\section{Results and discussion}

\subsection{Optimization of the multilayer nanoemulsion formulation}

The optimum lactoferrin concentration required to form the curcumin nanoemulsion composed of $5 \mathrm{wt} \%$ corn oil was determined (results not shown). A concentration of $2 \%$ was found to be the minimum amount of lactoferrin required to form stable nanoemulsions and, therefore, this concentration was used to prepare the nanoemulsions.

Regarding multilayer nanoemulsions, the biopolymer concentration used for their preparation must be carefully controlled to avoid particle aggregation (due to charge neutralization, bridging or depletion effects) (McClements, 2005). In order to establish the optimum alginate concentration required to prepare stable multilayer nanoemulsions, different alginate concentrations (from 0 to $0.3 \mathrm{wt} \%$ ) were tested and their effects on $\zeta$-potential and particle size were measured (Fig. 1).

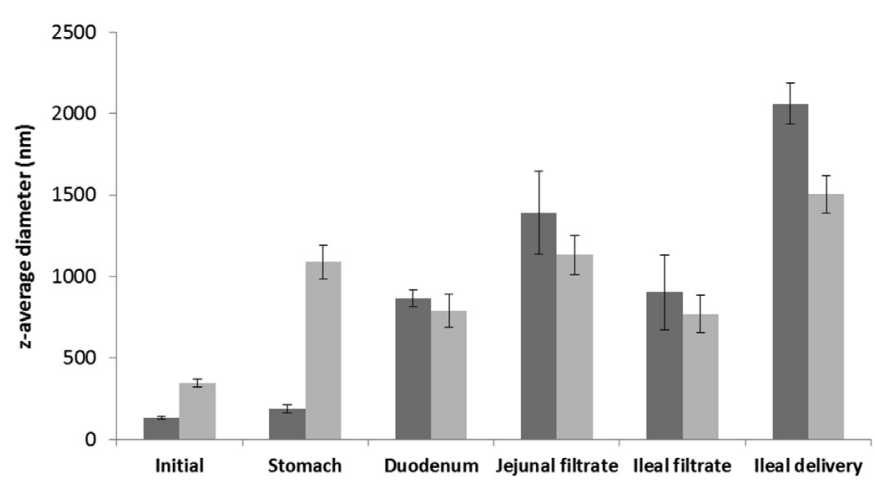

Fig. 2. Mean particle diameter of curcumin nanoemulsions stabilized by lactoferrin ( $\square$ ) and lactoferrin/alginate ( $\square$ ) as they pass through the dynamic in vitro digestion model
In the absence of alginate, lactoferrin-stabilized nanoemulsions exhibited a $\zeta$-potential of $27.47 \pm 1.11$ (at $\mathrm{pH} 4$ ). As the concentration of alginate increased, the $\zeta$-potential of the multilayer nanoemulsions became increasingly negative until it reached a constant value ( $\approx-30 \mathrm{mV}$ ), at an alginate concentration of $0.20 \%$ (Fig. 1a). This suggests that the anionic alginate molecules adsorbed to the surfaces of lactoferrin-stabilized nanoemulsions until they became saturated with alginate.

The increase in alginate concentration also influenced the particles' size (Fig. 1b). In the absence of alginate, lactoferrin-stabilized nanoemulsions exhibited a diameter of $149.3 \pm 4.9 \mathrm{~nm}$. At low alginate concentrations $(<0.06 \%)$, a significant increase in particle size occurred, suggesting extensive droplet aggregation which can be attributed to the low net charge on droplets as well as to bridging flocculation effects that occurred when droplet surfaces were not saturated with alginate (Li et al., 2010). At alginate concentrations higher than $0.08 \%$, an appreciable decrease in particles size was observed, suggesting that the emulsions were not susceptible to droplet aggregation at these alginate concentrations. This behaviour can be due to the strong electrostatic repulsion between the droplets and to the saturation of the droplets surfaces with alginate so that there was less bridging flocculation (Thanasukarn, Pongsawatmanit, \& McClements, 2006).

Therefore, $0.2 \%$ (representing a proportion of $10: 1$ of lactoferrin/ alginate) was the alginate concentration selected for the preparation of multilayer nanoemulsions, since, at this concentration, lactoferrin-stabilized nanoemulsions are completely coated by an alginate layer and there is no significant alginate excess in solution.

\subsection{In vitro digestion}

In vitro digestions were conducted in order to assess if the presence of a dietary fibre (in this case alginate) coating around lactoferrin-stabilized curcumin nanoemulsions influences their behaviour within a simulated GI tract.

\subsubsection{Influence of alginate on particle characteristics during in vitro digestion}

The effect of the alginate coating addition on particle size during in vitro digestion can be seen in Fig. 2. At stomach stage, the mean particle diameter of the nanoemulsions (stabilized by lactoferrin) remained relatively small, although some coalescence phenomena occurred (Fig. 3). On the contrary, the multilayer nanoemulsions (stabilized by lactoferrin and alginate) were highly unstable under gastric conditions, exhibiting a large increase in the particle size (indicating that extensive droplet aggregation occurred) and a high evidence of creaming (results not shown). This different behaviour is related to differences in their interfacial characteristics. At acidic 

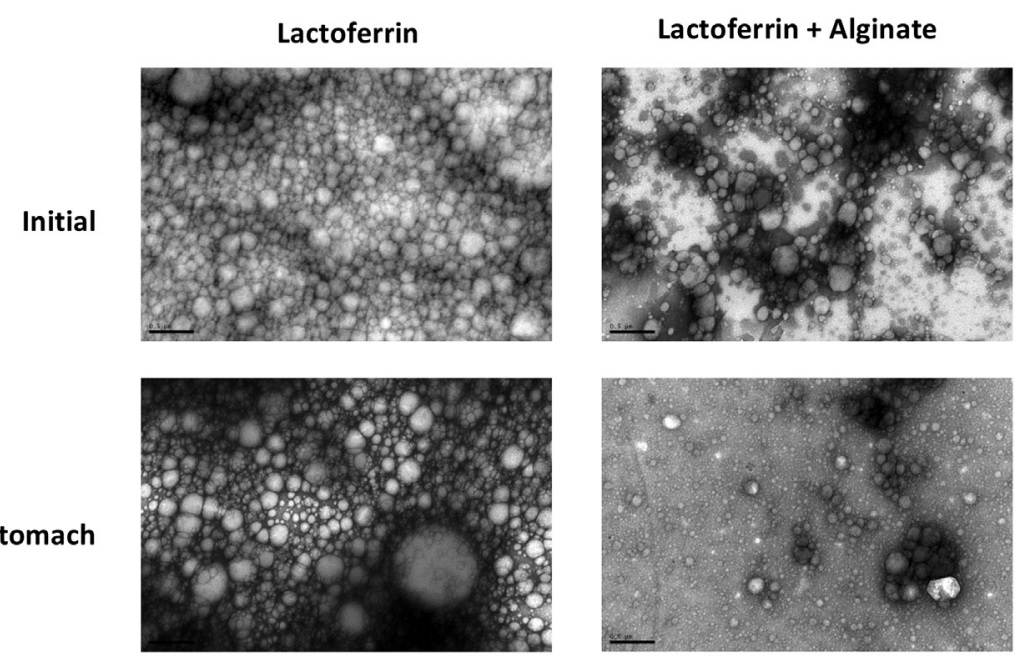

Stomach

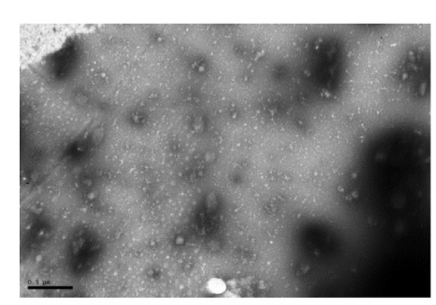

Duodenum
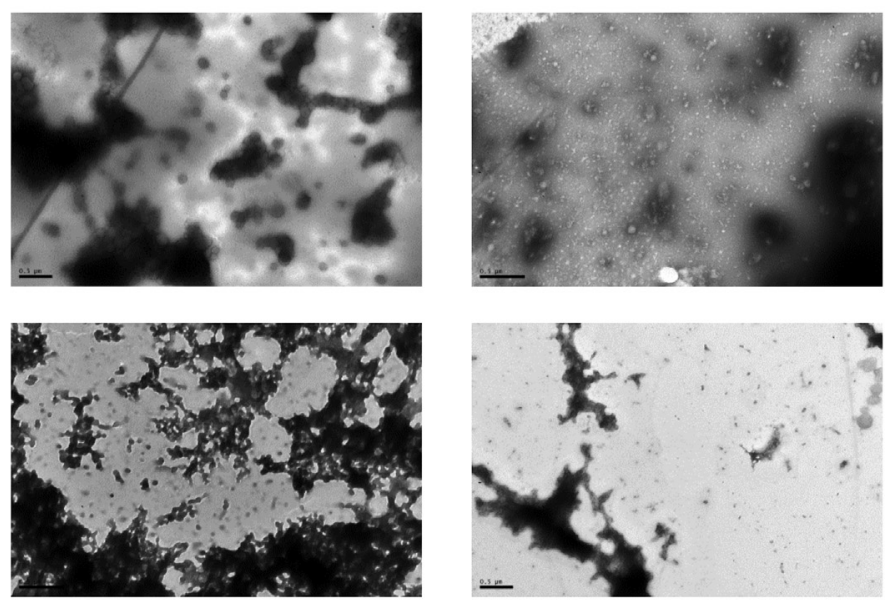

Jejunal Filtrate
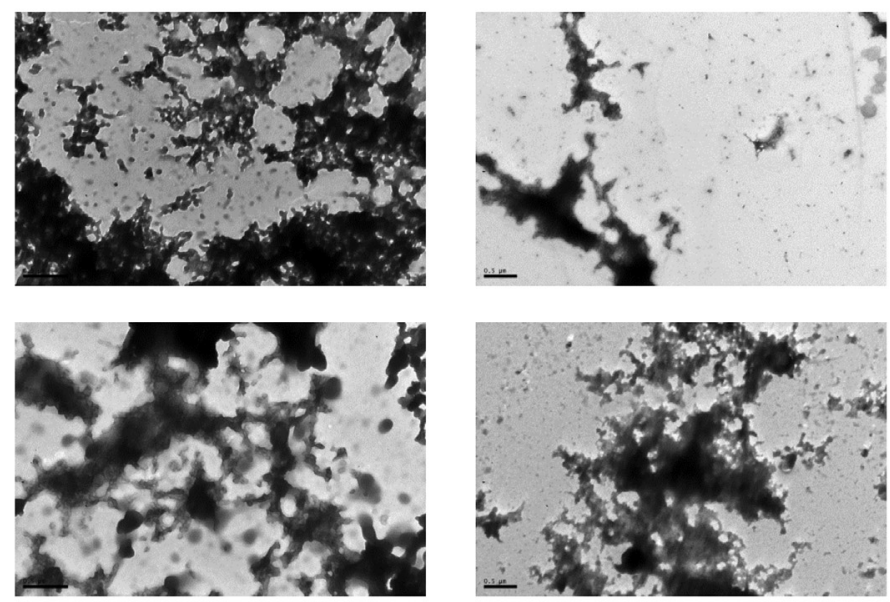

Ileal Filtrate
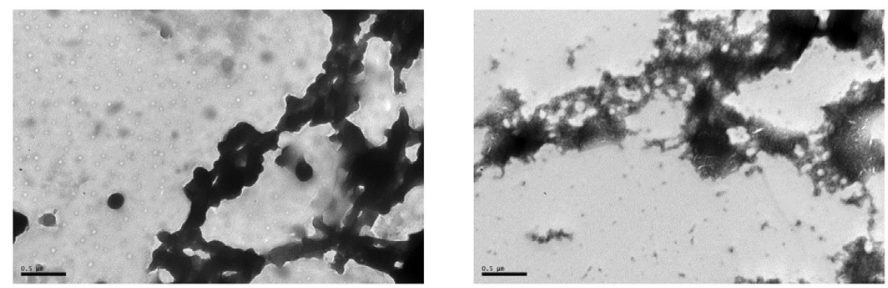

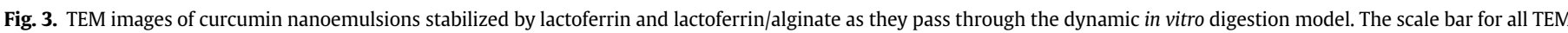
images is $0.5 \mu \mathrm{m}$.

conditions, lactoferrin-stabilized emulsions are highly positively charged (Fig. 4) and consequently there is a strong electrostatic repulsion between them, avoiding the emulsions' aggregation, coalescence and flocculation. On the other hand, alginate molecules have a linear anionic chain with no side chains, and therefore they are more susceptible to droplet aggregation through charge neutralization and bridging effects since there is little steric repulsion contribution (Tokle, Lesmes, Decker, \& McClements,
2012). Also, at acidic pH values, electrostatic attraction between alginate and the cationic lactoferrin-stabilized nanoemulsions is weakened because of the partial loss of negative charge on alginate below its pKa values. This could have led to bridging flocculation (i.e. a single alginate molecule may have been partially detached from one lipid droplet surface and became attached to another lipid droplet) (Tokle, Lesmes, \& McClements, 2010). Droplet aggregation of lactoferrin/alginate-stabilized nanoemulsions at the stomach 


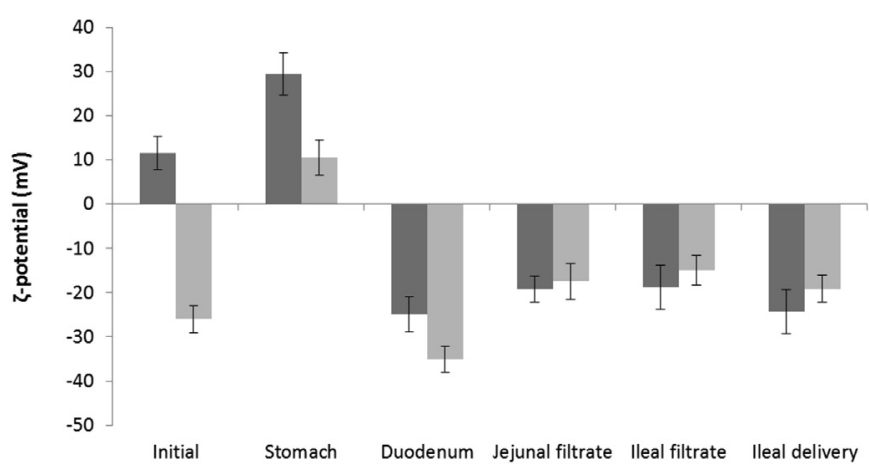

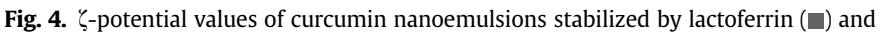
lactoferrin/alginate $(\square)$ as they pass through the dynamic in vitro digestion model.

stage is clearly visible in Fig. 3.

Under intestinal conditions, both nanoemulsions and multilayer nanoemulsions exhibited high particle diameters due to particle aggregation (flocculation and coalescence of emulsions) (Figs. 2 and 3 ). At these stages of digestion, bile salts may have been able to displace some lactoferrin from the lipid surface and lipase molecules were probably able to access and hydrolyse the lipids, producing free fatty acids (FFA), monoacylglycerides (MAG) and diacylgycerides (DAG) at the droplet surfaces. These surface-active products of digestion are ineffective at stabilizing oil-in-water emulsions against coalescence (Mun et al., 2007), which can explain the observed emulsions instability (i.e. high particle sizes).

As expected, jejunal and ileal filtrates (i.e. the fraction absorbed at jejunum and ileum compartments, respectively) exhibit particle sizes significantly $(p<0.05)$ lower than the ileal delivery (i.e. fraction that is not absorbed in the small intestine).

It should be noted that dynamic light scattering has a size limitation: it is only suitable for the measurement of particle diameters up to few microns, therefore, the results obtained for some samples should be analysed with care.

Regarding the effect of digestion on droplet charge (Fig. 4), it was observed that at stomach stage, the electrical charge of both emulsions became more positive which can be attributed to changes in solutions conditions ( $\mathrm{pH}$ and ionic strength), competitive displacement of the original emulsifier by surface active materials present in the gastric electrolyte solution, or adsorption of charged species from gastric electrolyte solution on top of the original emulsifier coating (Hur et al., 2009). In fact, regarding the multilayer nanoemulsion, the external alginate layer should be protonated at $\mathrm{pH} \approx 2$, not conferring charge to the nanoemulsions and thus can be more easily desorbed. In this way, the positive charge of lactoferrin can contribute to a higher positive $\zeta$-potential of multilayer nanoemulsions.

Once the gastric electrolyte solution contains pepsin, this protease may have hydrolysed the adsorbed lactoferrin molecules, thus facilitating their competitive displacement. Although, even if hydrolysis has occurred, the positively charged peptide should not have been displaced in a high extension, once at $\mathrm{pH} \approx 2$, the amine groups are all protonated, on the contrary of the initial solution, with higher $\mathrm{pH}$.

Comparatively with nanoemulsions stabilized only by lactoferrin, multilayer nanoemulsions exhibit a lower positive charge under gastric conditions, which suggests that, despite the fact that, at acid $\mathrm{pH}$, the electrostatic attraction between alginate and lactoferrin is weakened, alginate remained partly bound to lactoferrin-coated lipid droplets, thus protecting lactoferrin.

At duodenal conditions $(\mathrm{pH}=6.5)$ both lactoferrin and lactoferrin/alginate-stabilized nanoemulsions became highly negatively charged. If there were no changes in the interfacial composition, the lactoferrin-stabilized nanoemulsions would be slightly positively charged at neutral $\mathrm{pH}$, once the isoelectric point (pI) of lactoferrin is between 8.4 and 9.0 (Farnaud \& Evans, 2003). Therefore, the fact that the nanoemulsion has high negative charge suggests that there was a displacement of the lactoferrin layer from lipid surfaces by surface-active anionic molecules (e.g. free fatty acids, bile salts or lipase), the adsorption of anionic species (e.g. free fatty acids, bile salts or lipase) on top of the lactoferrin layer, or adsorption of small negatively charged ions from the small intestinal electrolyte solution (Tokle et al., 2012). There are no significant differences $(p>0.05)$ between $\zeta$-potential values of nanoemulsions and multilayer nanoemulsions at the subsequent stages of digestion process.

\subsubsection{Influence of alginate on lipid digestion}

The effect of alginate coating on free fatty acids release was also examined at the different stages of small intestine digestion (Fig. 5).

The overall extent of lipid digestion was fairly similar for both nanoemulsions and multilayer nanoemulsions, which suggests that alginate coating did not prevent lipid digestion. However, there are some differences in the percentage of FFA released in the different stages and fractions of small intestine digestion.

The major difference was observed in the jejunal filtrate fraction, with lactoferrin/alginate-stabilized nanoemulsions having a significantly $(p<0.05)$ lower amount of FFA as compared to lactoferrin-stabilized nanoemulsions. These results suggest that lactoferrin/alginate stabilized-nanoemulsions were digested at a slower rate than lactoferrin-stabilized nanoemulsions, which is in agreement with previous works (Tokle et al., 2012). Alginate is resistant to digestion in the stomach and small-intestine and has the ability to strongly bind calcium ions, preventing them from precipitating free fatty acids produced at the oil droplet surface $(\mathrm{Hu}$, Li, Decker, \& McClements, 2010). The decrease in the rate of lipid digestion in the presence of relatively small amounts of alginate has been thus mainly attributed to its ability to bind calcium ions. However, other physicochemical mechanisms may have occurred, e.g. presence of coating around the lipid droplets that prevented lipase from reaching their surface and binding multivalent cations required for lipase activity (Hu et al., 2010).

Another interesting result is that the total percentage of FFA absorbed in the small intestine (jejunum and ileum) is higher (although not significantly, $p>0.05$ ) for lactoferrin-stabilized nanoemulsions comparing to lactoferrin/alginate-stabilized

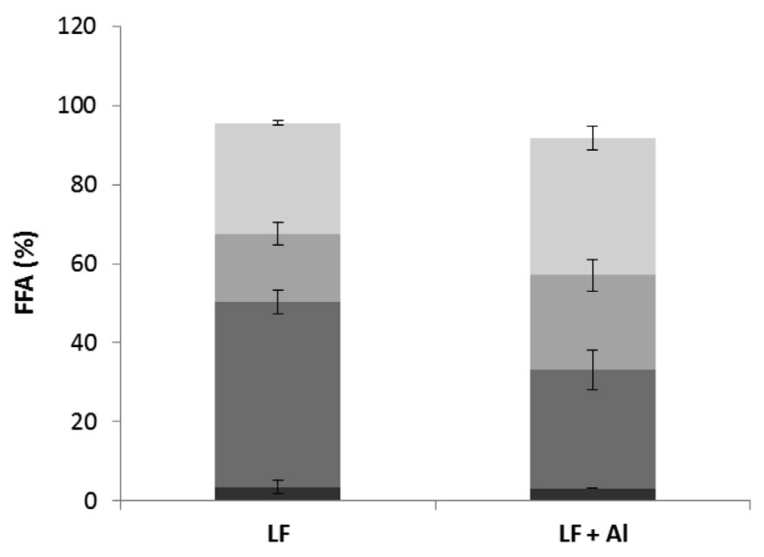

Fig. 5. Percentage of free fatty acids (FFA) released from lactoferrin (LF) and lactoferrin/alginate $(\mathrm{LF}+\mathrm{Al})$ stabilized nanoemulsions as they pass through the dynamic in vitro digestion model: Initial $(\square)$; Jejunal filtrate $(\square)$; Ileal filtrate $(\square)$ and Ileal delivery ( 
a)

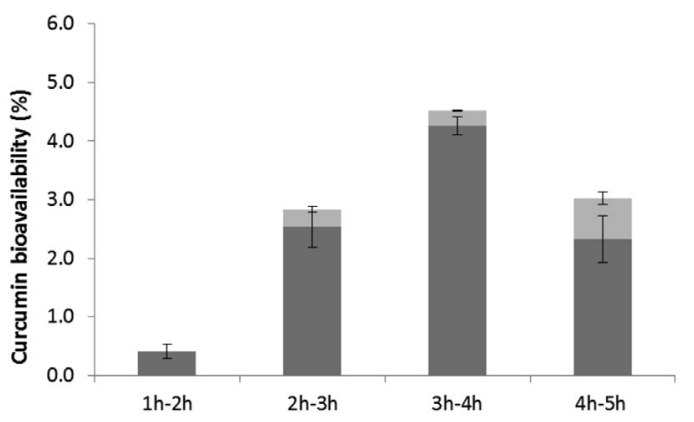

b)

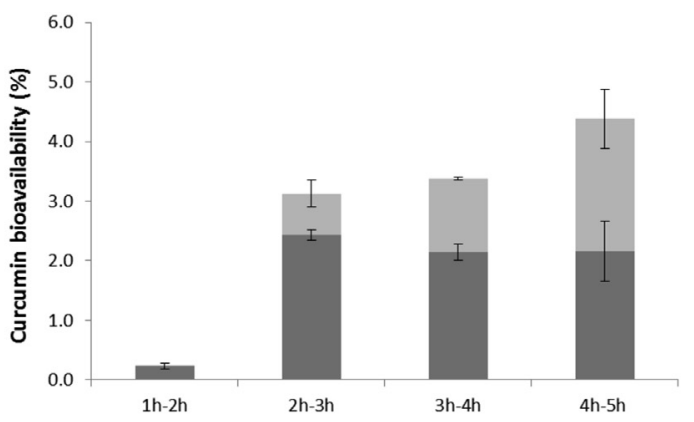

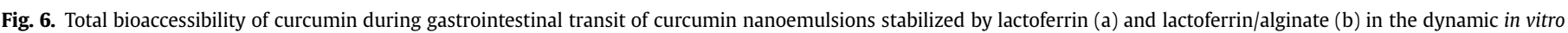

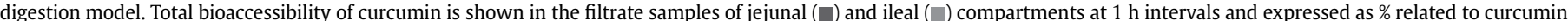
intake. The results are presented as the mean values of triplicate experiments.

nanoemulsions ( $64 \pm 6 \%$ and $54 \pm 11 \%$, respectively), which suggests that absorption of calories in the small-intestine may be reduced by addition of the alginate coating. This means that, although FFA were released almost in the same extent for both nanoemulsions and multilayer nanoemulsions ( $96 \pm 7$ and $92 \pm 12$, respectively), the presence of alginate hindered FFA absorption.

These results suggest that alginate coating may be useful to control the rate of lipid digestion and FFA adsorption within the gastrointestinal tract, but it does not prevent the adsorption of lipase to the lipid droplet and their consequent digestion. If the objective of the multilayer system is to increase the bioaccessibility of a water-insoluble bioactive compound (such as curcumin), it is necessary for the carrier oil surrounding them to be digested first, releasing the bioactive compounds so they can be taken up by mixed micelles (Tso, 2000).

\subsubsection{Influence of alginate on curcumin bioaccessibility}

Fig. 6 shows curcumin bioaccessibility, measured as curcumin concentration in the mixed micelle phase, in the filtrate samples of jejunal and ileal compartments as a function of time for nanoemulsions and multilayer nanoemulsions.

Curcumin bioaccessibility tends to increase with digestion time, and this behaviour can be attributed to the formation of digestion products that have the ability to form mixed micelles capable of solubilizing highly lipophilic components such as curcumin.

For both lactoferrin and lactoferrin/alginate-stabilized nanoemulsions the relative amount of curcumin was higher in the filtrate sample of jejunal compartment compared to the ileal compartment, however lactoferrin/alginate-stabilized nanoemulsions present a significantly $(p<0.05)$ higher curcumin bioaccessibility in ileal filtrate at all times. This result is in agreement with FFA release results (Fig. 5), i.e. for multilayer nanoemulsions the higher curcumin bioaccessibility observed in ileal filtrate was related with the higher amount of FFA found in this fraction. The access of lipases to encapsulated lipids is a critical step in determining the bioactivity of bioactive compounds, once the digestion products from TAG digestion (DG, MG, and FFA) may themselves become part of the mixed micelles, thereby increasing the overall solubilizing capacity of the small intestine, increasing the bioaccessibility and therefore the bioavailability of encapsulated bioactive compounds (Porter, Trevaskis, \& Charman, 2007). However, for both nanoemulsions, the total curcumin bioaccessibility is relatively low with most of the curcumin being recovered in the ileal deliveries and residues found adhered to the dynamic digestion model walls after the digestion was completed. These low values of curcumin bioaccessibility are quite surprising since previous works have reported higher curcumin bioaccessibility in oil- in-water emulsions stabilized also by proteins (Ahmed et al., 2012) or by surfactants (Pinheiro et al., 2013). A possible reason for this finding is that curcumin bound to lactoferrin molecules or digestion products of lactoferrin and therefore is not detected in the micelle phase. Similar results were obtained by other authors, for emulsions nanolaminated with lactoferrin and $\beta$-lactoglobulin coatings (Tokle, Mao, \& McClements, 2013). In this work, it has been suggested that lactoferrin is the responsible for the low $\beta$-carotene bioavailability of in a simulated GIT.

\section{Conclusions}

A dynamic digestion model comprising stomach, duodenum, jejunum and ileum was used to evaluate the impact of alginate coating on the digestion of curcumin nanoemulsions stabilized by lactoferrin. The interfacial characteristics of curcumin nanoemulsions had a significant impact on their physicochemical stability within the simulated GI tract: lactoferrin-stabilized nanoemulsions were stable in the stomach but aggregated in the small intestine, while lactoferrin/alginate-stabilized nanoemulsions were unstable under both gastric and small intestinal conditions. The overall extent of lipid digestion was fairly similar for both nanoemulsions and multilayer nanoemulsions, which suggests that alginate coating did not prevent lipid digestion. However, for lactoferrin-stabilized nanoemulsions the highest fraction of FFA was obtained in the jejunal filtrate whereas for lactoferrin/alginate-stabilized nanoemulsions the higher fraction of FFA was found in the ileal filtrate. Also, the higher curcumin bioaccessibility observed in the ileal filtrate of lactoferrin/alginatestabilized nanoemulsions is related with the higher amount of FFA found in this fraction. These results suggest that alginate coating may be instrumental in the control of the rate of lipid digestion and FFA adsorption within the GI tract, but the encapsulated lipid is digested at the same extent, releasing the lipophilic bioactive compound, which is then taken up by mixed micelles.

This work contributed to an improved understanding of how multilayer nanoemulsions behave within the human GI tract and this knowledge will be useful for the optimization of delivery systems that improve the physicochemical stability of emulsions in food products, while still releasing encapsulated lipophilic components in the GI tract.

\section{Acknowledgements}

The author Ana C. Pinheiro is recipient of a fellowship from the Fundação para a Ciência Tecnologia (FCT, Portugal) through grant SFRH/BPD/101181/2014. 
The authors thank the FCT Strategic Project of UID/BIO/04469/ 2013 unit, the project RECI/BBB-EBI/0179/2012 (FCOMP-01-0124FEDER-027462) and the project "BioInd - Biotechnology and Bioengineering for improved Industrial and Agro-Food processes", REF. NORTE-07-0124-FEDER-000028 Co-funded by the Programa Operacional Regional do Norte (ON.2 - O Novo Norte), QREN, FEDER. Thanks are also due to FCT for the financial support of the Research Unit 395 62/94-QOPNA (project PEst-C/QUI/UI0062/2013; FCOMP-01-0124-FEDER-037296).

\section{References}

Ahmed, K., Li, Y., McClements, D. J., \& Xiao, H. (2012). Nanoemulsion- and emulsionbased delivery systems for curcumin: encapsulation and release properties. Food Chemistry, 132(2), 799-807.

Balcão, V. M., Costa, C. I., Matos, C. M., Moutinho, C. G., Amorim, M., Pintado, M. E. et al. (2013). Nanoencapsulation of bovine lactoferrin for food and biopharmaceutical applications. Food Hydrocolloids, 32(2), 425-431.

Decher, G., \& Schlenoff, J. B. (2003). Polyelectrolyte multilayers, an overview. Weinheim Wiley-VCH Verlag GmbH \& Co (KGaA).

Farnaud, S., \& Evans, R. W. (2003). Lactoferrin-a multifunctional protein with antimicrobial properties. Molecular Immunology, 40(7), 395-405.

Hu, M., Li, Y., Decker, E. A., \& McClements, D. J. (2010). Role of calcium and calciumbinding agents on the lipase digestibility of emulsified lipids using an in vitro digestion model. Food Hydrocolloids, 24(8), 719-725.

Hur, S. J., Decker, E. A., \& McClements, D. J. (2009). Influence of initial emulsifier type on microstructural changes occurring in emulsified lipids during in vitro digestion. Food Chemistry, 114(1), 253-262.

Lee, K. Y., \& Mooney, D. J. (2012). Alginate: properties and biomedical applications. Progress in Polymer Science, 37(1), 106-126.

Li, Y., Hu, M., Du, Y., Xiao, H., \& McClements, D. J. (2011). Control of lipase digestibility of emulsified lipids by encapsulation within calcium alginate beads. Food Hydrocolloids, 25(1), 122-130.

Li, Y., Hu, M., Xiao, H., Du, Y., Decker, E. A., \& McClements, D. J. (2010). Controlling the functional performance of emulsion-based delivery systems using multicomponent biopolymer coatings. European Journal of Pharmaceutics and Biopharmaceutics, 76(1), 38-47.

Maurstad, G., Mørch, Y. A., Bausch, A. R., \& Stokke, B. T. (2008). Polyelectrolyte layer interpenetration and swelling of alginate-chitosan multilayers studied by dua wavelength reflection interference contrast microscopy. Carbohydrate Polymers, 71(4), 672-681.

McClements, D. J. (2005). Theoretical analysis of factors affecting the formation and stability of multilayered colloidal dispersions. Langmuir, 21(21), 9777-9785.

McClements, D. J. (2010). Design of nano-laminated coatings to control bioavailability of lipophilic food components. Journal of Food Science, 75(1), R30-R42.

McClements, D. J. (2013). Edible lipid nanoparticles: digestion, absorption, and potential toxicity. Progress in Lipid Research, 52(4), 409-423.
McClements, D. J., \& Xiao, H. (2012). Potential biological fate of ingested nanoemulsions: influence of particle characteristics. Food \& Function, 3(3), 202-220.

Minekus, M., Jelier, M., Xiao, J.-Z., Kondo, S., Iwatsuki, K., Kokubo, S., et al. (2005). Effect of partially hydrolyzed guar gum (PHGG) on the bioaccessibility of fat and cholesterol. Bioscience, Biotechnology, and Biochemistry, 69(5), 932-938.

Mun, S., Decker, E. A., \& McClements, D. J. (2007). Influence of emulsifier type on in vitro digestibility of lipid droplets by pancreatic lipase. Food Research International, 40(6), 770-781.

Pinheiro, A. C. Lad, M., Silva, H. D., Coimbra, M. A., Boland, M., \& Vicente, A. A. (2013). Unravelling the behaviour of curcumin nanoemulsions during in vitro digestion: effect of the surface charge. Soft Matter, 9(11), 3147-3154.

Pinsirodom, P., \& P, K. L. (2005). Lipase assays. In R. E. Wrolstad (Ed.), Handbook of food analytical chemistry (pp. 371-383). Hoboken, NJ: Wiley.

Porter, C. J. H., Trevaskis, N. L., \& Charman, W. N. (2007). Lipids and lipid-based formulations: optimizing the oral delivery of lipophilic drugs. Nature Reviews Drug Discovery, 6, 231-248.

Reis, P., Raab, T., Chuat, J., Leser, M., Miller, R., Watzke, H., et al. (2008). Influence of surfactants on lipase fat digestion in a model gastro-intestinal system. Food Biophysics, 3(4), 370-381.

Salminen, H., Herrmann, K., \& Weiss, J. (2013). Oil-in-water emulsions as a delivery system for n-3 fatty acids in meat products. Meat Science, 93(3), 659-667.

Salvia-Trujillo, L., Qian, C., Martín-Belloso, O., \& McClements, D. J. (2013). Influence of particle size on lipid digestion and $\beta$-carotene bioaccessibility in emulsions and nanoemulsions. Food Chemistry, 141(2), 1472-1480.

Shimoni, G., Shani Levi, C., Levi Tal, S., \& Lesmes, U. (2013). Emulsions stabilization by lactoferrin nano-particles under in vitro digestion conditions. Food Hydrocolloids, 33(2), 264-272.

Thanasukarn, P., Pongsawatmanit, R., \& McClements, D. J. (2006). Utilization of layer-by-layer interfacial deposition technique to improve freeze-thaw stability of oil-in-water emulsions. Food Research International, 39(6), 721-729.

Tokle, T., Lesmes, U., Decker, E. A., \& McClements, D. J. (2012). Impact of dietary fiber coatings on behavior of protein-stabilized lipid droplets under simulated gastrointestinal conditions. Food \& Function, 3(1), 58-66.

Tokle, T., Lesmes, U., \& McClements, D. J. (2010). Impact of electrostatic deposition of anionic polysaccharides on the stability of oil droplets coated by lactoferrin. Journal of Agricultural and Food Chemistry, 58(17), 9825-9832.

Tokle, T., Mao, Y., \& McClements, D. (2013). Potential biological fate of emulsionbased delivery systems: lipid particles nanolaminated with lactoferrin and $\beta$ lactoglobulin coatings. Pharmaceutical Research, 30(12), 3200-3213.

Tokle, T., \& McClements, D. J. (2011). Physicochemical properties of lactoferrin stabilized oil-in-water emulsions: effects of pH, salt and heating. Food Hydrocolloids, 25(5), 976-982.

Tso, P. (2000). Overview of digestion and absorption. In M. H. S. K. D. Crissinger (Ed.), Biochemical and physiological aspects of human nutrition (pp. 75-90). Philadelphia: W.B. Saunders Co.

Zhou, J., Romero, G., Rojas, E., Ma, L., Moya, S., \& Gao, C. (2010). Layer by layer chitosan/alginate coatings on poly(lactide-co-glycolide) nanoparticles for antifouling protection and folic acid binding to achieve selective cell targeting. Journal of Colloid and Interface Science, 345(2), 241-247. 